\title{
Sistematización del trabajo que realiza el Centro Familiar para la Integración y el Crecimiento en la prevención y atención a víctimas de violencia
}

\author{
Systematization of the Work Performed by the Family \\ Center for Integration and Growth in the Prevention \\ and Attention to Victims of Violence
}

Celia Mancillas Bazán*

\begin{abstract}
RESUMEN
El objetivo del presente estudio es analizar la pertinencia de la sistematización de la práctica desde la narrativa y la horizontalidad para comprender cómo una asociación civil construye alternativas de apoyo a personas y familias en condiciones de vulnerabilidad, especialmente a aquellos que han sido víctimas de violencia. Se parte de la comprensión de seis dimensiones: procesos de duelo, resiliencia comunitaria, educación para la paz, prácticas restaurativas, prevención del suicidio y desarrollo humano y espiritualidad ignaciana, para favorecer la comprensión de los procesos de cambio psicosociales en un contexto caracterizado por la pobreza y la violencia. El estudio se llevó a cabo en el Centro Familiar para la Integración y el Crecimiento, A.C. en Ciudad Juárez, Chihuahua. Los participantes en el estudio fueron el grupo de trabajo del mencionado Centro, los beneficiarios y los aliados del mismo. Se emplearon técnicas cualitativas como: relatos de vida, relatos testimoniales, entrevistas en profundidad y grupos de discusión.
\end{abstract}

Palabras clave: procesos de duelo, resiliencia comunitaria, educación para la paz, prácticas restaurativas, prevención del suicidio, desarrollo humano, espiritualidad ignaciana

\section{ABSTRACT}

The aim of the present study is to analyze the relevance of the systematization of practice from narrative and horizontality in order to understand how a civil association (CA) builds aid alternatives for vulnerable people and families, especially those victims of violence. Based on the understanding of six dimensions - grieving processes, community resilience, peace education, restorative practices, suicide prevention, human development and Ignatian spirituality - it is favored the understanding of the psychosocial change processes in a context characterized by poverty and violence. The study was led in the CA Family Center for Integration and Growth in Ciudad Juarez, Chihuahua. The participants in the study were the work group of the aforementioned Center, its beneficiaries and its allies. The qualitative techniques used were life stories, testimonials, in-depth interviews and group discussions.

Key words: grieving processes, community resilience, peace education, restorative practices and suicide prevention, human development and Ignatian Spirituality.

\footnotetext{
*Universidad Iberoamericana; celia.mancillas@ibero.mx. Agradecemos el apoyo y financiamiento otorgado por el Instituto de Investigaciones para el Desarrollo con Equidad de la Universidad Iberoamericana.
} 


\section{INTRODUCCIÓN}

La violencia y los conflictos entre individuos, grupos sociales y comunidades son una amenaza especialmente poderosa a la salud mental (OMS, 2004). Las consecuencias de las situaciones de violencia perjudican directamente el bienestar subjetivo de la población afectada. La prevención de la violencia atañe a la sociedad en su conjunto, pero existen esfuerzos de grupos sociales organizados que buscan de manera activa aminorar el impacto de este fenómeno a través de la implementación de estrategias preventivas específicas y de atención a las personas víctimas de violencia, tratando de favorecer una cultura de paz frente a la creciente cultura de violencia.

Ciudad Juárez, en la frontera norte de México, es una ciudad receptora de migrantes que tuvo un crecimiento demográfico acelerado durante la mayor parte del siglo pasado. Entre 1940 y 2010 su población pasó de 55024 habitantes a 1332131 (Martínez, 2012). Tal crecimiento y el impulso de un proyecto económico que privilegió la oferta de empleo y desatendió los aspectos básicos del desarrollo humano y social, generaron diversos tipos de violencia, visibles en dos problemáticas que han hecho que la ciudad haya sido identificada durante varios años como "la más violenta del mundo": los asesinatos de mujeres (feminicidios) y los niveles más altos de homicidios dolosos a nivel mundial.

Ciudad Juárez tuvo una crisis sin precedentes de violencia entre 2008 y 2011. De acuerdo a Lima y Lima (2014, p. 83), se registraron 9794 homicidios en el Municipio de Juárez. Esparza, Gutiérrez, Montañez y Carrillo (2016) señalan que uno de los elementos más difíciles del tipo de violencia que se ha experimentado en Ciudad Juárez es su carácter de impredecible; esto quiere decir que, para antes de 2008, aunque existían diversas manifestaciones de violencia, ésta se encontraba focalizada, lo que permitía que los ciudadanos identificaran, de manera predecible, en qué lugares y momentos del día podían correr peligro. Sin embargo, para después de este ańo, las ejecuciones, así como cualquier tipo de acto violento podían suceder sin distinción del lugar o la hora del día. 
Si bien dicha situación se identifica como un grave problema de seguridad pública, poco se han reconocido los daños psicoafectivos y sociales en la población. En consecuencia, los programas orientados a la atención de los mismos han sido escasos y limitados y han llegado poco a las poblaciones más afectadas. Han sido, en su mayoría, las organizaciones de la sociedad civil quienes han desarrollado programas para la atención de la violencia y sus secuelas, a través de acciones dirigidas a la atención del estrés postraumático, duelo-tanatología, proyectos comunitarios de construcción de redes para la seguridad ciudadana, entre otros.

El Centro Familiar para la Integración y el Crecimiento, A.C. (CFIC) es una organización en Ciudad Juárez que ha creado una serie de estrategias de intervención para el acompañamiento a víctimas de violencia, así como de prevención a través de favorecer una educación para la paz en adultos, adolescentes y niños. Este Centro busca contribuir a generar procesos de cambio para lograr un mejor funcionamiento de las personas, las familias y la comunidad, con el fin de incrementar una mejor calidad de vida y manejo de situaciones de conflicto, especialmente en condiciones de vulnerabilidad. Esta asociación civil se fundó en febrero de 1999, desde la comunidad de la Parroquia del Señor de la Misericordia. Hasta hoy han atendido alrededor de 46800 personas a través de distintos tipos de diplomados y talleres organizados en tres áreas: tanatología, psicoterapia y desarrollo humano (CFIC, 2016).

\section{SISTEMATIZACIÓN DE BUENAS PRÁCTICAS Y EXPERIENCIAS DESDE EL DIÁLOGO Y LA HORIZONTALIDAD}

La sistematización de prácticas significativas del trabajo del CFIC tiene como antecedente el Diplomado de Desarrollo Humano y Espiritualidad Ignaciana en el que colaboran la Universidad Iberoamericana y el Centro, que comenzó en otoño de 2015 y que continúa a la fecha. De ahí surgió un interés por recuperar y dar cuenta de prácticas valiosas de las que se derivan aprendizajes importantes que pueden ayudar a enriquecer la práctica de ésta, otras asociaciones civiles de Ciudad Juárez y de otros contextos que tuvieran en su quehacer la prevención y atención de 
personas víctimas de violencia. En 2017 acordamos desarrollar, en conjunto, una sistematización de las prácticas significativas de esta asociación civil para recuperar saberes y dar cuenta de sus estrategias, prácticas y experiencias, vinculándolas con el contexto social, político y cultural de Ciudad Juárez, a fin de crear condiciones para la reflexión de su quehacer y para facilitar procesos de transformación social y cultural a partir de develar situaciones y abordar alternativas.

La sistematización forma parte de la educación popular que fue creada en América Latina y que se fundamenta en el pensamiento de Paulo Freire y otras corrientes, quienes consideran este abordaje como una investigación acción participativa (IAP) (Crispín y Ruiz, 2010). La IAP es un conjunto de principios y procedimientos metodológicos que permite generar conocimientos colectivos sobre una determinada realidad social (Rodríguez, Gil y García, 1999), a través de la cual se establece una colaboración directa con los actores principales para crear una construcción colectiva del conocimiento sobre sus prácticas.

La sistematización tiene varias concepciones. De acuerdo con Jara (1998), es aquella interpretación crítica de una o varias experiencias que, a partir de su ordenamiento y reconstrucción, descubre o explicita la lógica del proceso vivido, los factores que han intervenido en dicho proceso, cómo se han relacionado entre sí, y por qué lo han hecho de ese modo.

Para Barnechea y Morgan (2010), la sistematización es una forma de extraer y de comunicar los conocimientos que se producen en proyectos de intervención en la realidad, con la intencionalidad de transformación. Es una manera distinta de investigar la realidad social para aportar al conocimiento científico que tiene lugar en el marco de proyectos y programas de desarrollo, esto es, de intervenciones intencionadas con objetivos de transformación de la realidad.

De acuerdo con el Programa de las Naciones Unidas para el Desarrollo (PNUD, 2017, p. 11), "la sistematización es el proceso para identificar, capturar y documentar aprendizajes y experiencias claves de uno o varios proyectos con el propósito de transferir y adaptar el conocimiento". Implica un análisis profundo de cómo fue posible lograr lo que se logró, lo que funcionó, factores 
claves de éxito, alternativas de acción y recomendaciones derivadas de la práctica.

De Souza (2008) sitúa la sistematización como una modalidad especial de investigación social, específicamente educativa, que se constituye como un instrumento didáctico. La sistematización, afirma:

Se configura de procesos que se concretizan en la actividad sistematizadora a lo largo de las intervenciones institucionales que posibilita, que los sujetos de una acción social y/o colectiva se apropien de su propia experiencia por la construcción del sentido de su vivencia en los programas de desarrollo, lo cual puede ser extendido a la existencia histórica (De Souza, 2008, p. 10).

Sostiene que las actividades de los programas comunitarios son procesos aunados a significaciones (significados y sentidos) intersubjetivamente producidas y compartidas por sus participantes. Para Martinic (como se citó en De Souza, 2008), los hechos sociales, siendo distintos de los naturales, obligan a colocar de otro modo la relación sujeto-objeto en la construcción de conocimientos, y esto implica que más que descubrir las leyes objetivas del comportamiento, lo que interesa es producir esquemas de interpretación sobre lo real que permitan comprender y descifrar las acciones sociales.

El término "buenas prácticas" ha sido definido por la Organización de las Naciones Unidas para la Alimentación y la Agricultura (FAO, 2015, p. 1):

Una buena práctica no es tan sólo una práctica que se define buena en sí misma, sino que es una práctica que se ha demostrado que funciona bien y produce buenos resultados, y, por lo tanto, se recomienda como modelo. Se trata de una experiencia exitosa, que ha sido probada y validada, en un sentido amplio, que se ha repetido y que merece ser compartida con el fin de ser adoptada por el mayor número posible de personas.

También describen los criterios que permiten la identificación de buenas prácticas: 1) que sea efectiva y exitosa, esto es, que sea un medio eficaz para el logro de los objetivos planteados y 
que se demuestre que tiene un impacto positivo en los individuos y en las comunidades; 2) que es sostenible desde una perspectiva ambiental, económica y social; esto es, que cubra las necesidades inmediatas, particularmente de los más pobres, sin que esto comprometa su capacidad para hacer frente a las necesidades futuras; 3) sensible a los asuntos de género, comprendiendo cómo los hombres y mujeres participan en los procesos; 4) técnicamente posible; esto es, que la viabilidad técnica sea fácil de aprender y aplicar; 5) es el resultado de un proceso participativo, ya que éste favorece un sentido de pertenencia de las decisiones y de las acciones; 6) que sea replicable y adaptable; es decir, que tenga potencial de repetición, y 7) que reduzca los riesgos de desastres/ crisis, esto es, que contribuya a la reducción de los riesgos de desastres/crisis para la resiliencia (FAO, 2015).

"Buenas prácticas" o "experiencias significativas" son dos concepciones que han sido analizadas por Pieck (2012), quien destaca la importancia de sistematizar las experiencias vividas, esto es, lo que las personas piensan de eso que les sucede, dejando que esa experiencia se presente y se haga relato. Lo significativo, sostiene:

Tiene que ver con la medida de respuesta a las necesidades, con la adecuación a los contextos, con la satisfacción de estudiantes y profesores, con el propio significado que tiene la experiencia, con los vínculos que se establecen, con las relaciones personales que se generan, con la forma de enfrentar las adversidades y los retos, con la propia innovación y la propuesta de modelos alternativos, con los procesos desencadenados en los actores (estudiantes, padres de familia, autoridades y maestros), con el grado de solidaridad y compromiso, con la historia misma del programa (Pieck, 2012, p. 42).

Las bases epistemológicas del proceso de la sistematización fueron analizadas por Ghiso (1998), quien identificó, entre ellas, el enfoque dialógico e interactivo, a través del cual se entienden las experiencias como espacios de interacción, comunicación y relación y donde se construye el conocimiento a partir de los referentes externos e internos que permiten analizar las áreas problemáticas expresadas en los procesos conversacionales que se dan 
en toda práctica social. Sostiene que es necesario reconocer toda acción como un espacio dialógico y relacionar el diálogo con el contexto, reconociendo cómo se organiza, coordina y condiciona la interacción.

Para Corona y Kaltmeir (2012) el diálogo es un fenómeno social en el que los sujetos se construyen de forma permanente a partir de las relaciones con los otros. De esta manera, sostiene, el diálogo es un proceso horizontal más amplio que pone en cuestión las normas, los saberes y las prácticas institucionalizadas. La construcción del diálogo se yergue desde las diferencias, las polifonías y las hibridaciones e implica una apertura al otro y el deseo de conocerlo, como también entrar a un proceso de reconocerse a sí mismo. Así, en un proceso de investigación que se basa en los principios de horizontalidad, reciprocidad y dialogicidad, se supone que el "investigador" y el "investigado" llegan a una nueva mirada.

Los ejes rectores de este trabajo de sistematización, basados en los planteamientos anteriores, son los siguientes: 1) es un proceso de producción del conocimiento colaborativo basado en la reflexividad dialógica y la equidad como condiciones centrales, donde los interactuantes se construyen en forma permanente; 2) es un proceso de reflexión crítica, donde importan los procesos de construcción de las prácticas, los resultados de éxito y los aspectos problemáticos o contradictorios que se presenten en las experiencias y en la trama de la vida cotidiana; 3) resulta de la integración de los distintos saberes asociados con las prácticas, experiencias y significados asociados, así como aspectos psicoafectivos y relacionales; 4) es un proceso metodológico cualitativo y participativo que permite dar cuenta de las experiencias de intervención en determinada realidad social; 5) es un proceso que favorece la comprensión y la reflexión de un equipo sobre su propio trabajo y de los participantes en la sistematización, de manera que les permite explicitar procesos y relaciones $y$, por último, 6) es un proceso que busca generar conocimientos desde la práctica, que puedan contribuir a la teoría y con ello a la transformación de la realidad.

Cabe mencionar que un eje transversal de análisis de este estudio es el género ya que se asocia diferencialmente a la experiencia de vulnerabilidad, de factores de riesgo y de estrategias 
de afrontamiento, entre otros aspectos, en función del contexto social y cultural en el que se desarrollan. De acuerdo con Palomar (2015), la socialización diferencial de acuerdo con el género lleva al desarrollo o no de ciertas capacidades en hombres y mujeres que se vinculan, por ejemplo, con la resiliencia, o en la manifestación diferencial de la violencia de género en hombres y mujeres, o en las formas de afrontamiento.

\section{PERSPECTIVA MULTIDIMENSIONAL EN LA ATENCIÓN A PERSONAS VÍCTIMAS DE VIOLENCIA}

En sus casi 20 años de existencia, el CFIC ha generado una serie de estrategias para dar respuesta a necesidades emergentes de apoyo a personas, familias y distintos grupos sociales en condiciones de vulnerabilidad, especialmente a aquellos que han sido víctimas de violencia en el contexto de Ciudad Juárez. Esto ha creado una extensa producción de cursos, talleres, diplomados, procesos terapéuticos y otras actividades. La elección de ¿qué queremos sistematizar? nos condujo, inicialmente, a tratar de comprender cuáles eran los principales ejes que organizaban las distintas prácticas de prevención y de intervención del CFIC.

Primer eje: procesos de duelo y pérdida. Éstas fueron las primeras prácticas que surgieron en el CFIC para atender a las personas y familiares que habían sufrido pérdidas en distintas circunstancias, generalmente vinculadas con la violencia. De acuerdo a Meza, García, Torres, Castillo, Sauri y Martínez (2008), el duelo es una reacción emocional y de la conducta que se manifiesta como sufrimiento y aflicción cuando se rompe un vínculo afectivo. El duelo tiene componentes psicológicos, físicos y sociales y su intensidad y duración son proporcionales al afecto (apego) y a la pérdida, siendo que la intensidad del duelo no depende de la naturaleza del objeto perdido, sino del valor atribuido al mismo. El duelo no se asocia únicamente a la muerte de un ser querido, sino también a la pérdida de alguna otra abstracción como la libertad, el ideal, la seguridad, etcétera.

En general, se considera que la vivencia de una persona que ha enfrentado la muerte de un familiar por una situación violenta y traumática, como lo es el homicidio, es cualitativamente dife- 
rente a lo que viven otras personas que enfrentan, por ejemplo, duelos anticipados. Esto es, cuando un miembro de la familia o un ser querido ha sido diagnosticado con alguna enfermedad terminal, las personas realizan un proceso de duelo diferente a cuando la muerte es súbita y, por tanto, inesperada. Además, de acuerdo a Armour (2003), el hecho de que las condiciones que rodean a la muerte sean traumáticas, dificulta la resignificación de quienes permanecen con vida, ya que se enfrentan a hechos sin sentido y carentes de razón. Esta autora cuestiona fuertemente las ideas de los modelos que mencionan que las personas avanzan (o sanan) cuando logran dar un significado distinto a aquella pérdida que han experimentado. Finalmente, concluye, esa "resignificación" aparece en las personas que han experimentado la muerte violenta de un familiar, de la siguiente manera: "una búsqueda intensa de aquello que importa". Además, esta resignificación toma forma en las acciones, por ejemplo: "luchar por aquello que se considera bueno o está bien" (que puede manifestarse en forma de activismo), o bien vivir la vida honrando la memoria de aquella persona que ya no está, usar la experiencia propia para ayudar a otras personas y tratar de buscar intensamente, un "aprendizaje positivo" de la experiencia traumática.

Gillies y Neimeyer (2006), a partir de una revisión de investigaciones cuantitativas, cualitativas y mixtas, proponen un modelo que analiza las vías de reconstrucción de significado en respuesta a la pérdida de un ser querido. Sostienen que el sistema de creencias de una persona (sobre la propia muerte), previo a la muerte de un familiar, influye de manera determinante sobre la experiencia que tenga del proceso, incluso independientemente del tipo de fallecimiento que haya sucedido. Asimismo, definen que los procesos clave de creación de significado propuestos en el modelo son: la creación de sentido, la búsqueda de beneficios y el cambio de identidad. Mediante estos procesos, las personas en duelo participan en una reconstrucción de significados, a través de la cual las estructuras de sentido previo a la pérdida pueden ser revisadas, reevaluadas, renovadas y/o reconstruidas. De acuerdo con el modelo, el proceso de reconstrucción produce nuevas estructuras de significado, posteriores a la pérdida, a través de las cuales los deudos llegan a ver el mundo de una nueva manera. 
Segundo eje: resiliencia comunitaria. Una buena parte de las personas están expuestas a situaciones de violencia o amenaza a lo largo de su vida y afrontan esas situaciones de maneras distintas. Así, algunas tienen serias dificultades para sobreponerse ante situaciones de estrés, y otras, en cambio, ante situaciones muy adversas, logran sobreponerse y aun salir fortalecidos. A estos individuos se les llama resilientes. Para Uriarte (2013), la resiliencia comunitaria se refiere a la capacidad del sistema social y de las instituciones para hacer frente a las adversidades y para reorganizarse, posteriormente, de modo que mejoren sus funciones, su estructura y su identidad. Comprende tanto los recursos tangibles, es decir los recursos materiales, humanos o procedimentales que protegen a los individuos y compensan las debilidades, como los intangibles, esto es, aquellos que capacitan para sobreponerse a las dificultades y para lograr una adaptación exitosa. La resiliencia comunitaria, para Twigg (2007), no es una intervención específica o delimitada a responder a un determinado acontecimiento adverso, sino que es necesario comprenderla como la capacidad de la comunidad de detectar y prevenir adversidades, la capacidad de absorción de una adversidad impactante y la capacidad para recuperarse tras un dańo.

Para Madsen y O'Mullan (2016), si se compara con el estudio de la resiliencia individual, el campo de la resiliencia comunitaria es de reciente creación. Los autores realizaron un estudio cualitativo sobre resiliencia comunitaria en una población rural australiana. Los resultados de su estudio indican que la resiliencia comunitaria se compone de los siguientes elementos: conexión social, aceptación optimista, el aprendizaje de la tolerancia y la paciencia y el aprendizaje del pasado para las generaciones futuras. Sostienen que la conexión social es uno de los elementos más importantes que forman la resiliencia comunitaria, ya que favorece que las relaciones entre las personas que forman parte de una comunidad sean adecuadas, cercanas y tan fuertes como para soportar experiencias potencialmente traumáticas, como puede ser un desastre natural. Afirman que el capital social forma una parte vital de la resiliencia comunitaria y que, aunque dicha resiliencia se pone a prueba únicamente durante los tiempos de adversidad, 
el capital social necesita ser desarrollado con anticipación a dichos desastres.

Dreyer (2015) analiza la resiliencia comunitaria y la espiritualidad en el periodo posterior al apartheid en Sudáfrica. Plantea que la resiliencia comunitaria es resultado de un proceso de restauración que es posterior a la experiencia de una comunidad por haber enfrentado un trauma colectivo. Los traumas colectivos dañan el tejido social y el sentido de comunidad de las personas. A menudo, éstos son causados por un evento inesperado y devastador que prolonga sus efectos en el tiempo. El trauma social significa una traición a la confianza de las personas, situación que provoca que se sientan devaluadas y humilladas y con una constante sensación de pérdida. Todo lo que hasta el momento del trauma habían creído sobre los otros y sobre la vida, se derrumba. Por tanto, el proceso de recuperación, así como el trauma, debe ser colectivo e implicar la actuación de diferentes actores sociales.

Tercer eje: educación para la paz. La magnitud de la violencia que se ha vivido en Ciudad Juárez y sus niveles de gravedad han obligado a desarrollar respuestas desde un modelo basado en la educación para la paz, centrado en la solución no violenta de conflictos. Las prácticas del CFIC se basan fundamentalmente en las Escuelas de Perdón y Reconciliación (ES.PE.RE), creadas por Leonel Narváez Gómez, como una alternativa pedagógica de la educación emocional (Escobedo, 2014). Ésta tiene el propósito de aportar a la restauración de las relaciones familiares y comunitarias afectadas por el aumento de la violencia intrafamiliar y social, mediante la oferta de espacios formativos y vivenciales en pedagogías de convivencia y favorecer el empoderamiento personal, familiar y comunitario.

Álvarez y González (2013) mencionan que, cuando la violencia está normalizada, es necesario generar procesos de construcción de la paz, lo que requiere de formación y entendimiento que no sólo corresponde a los trabajadores sociales, a los pedagogos y a los psicólogos, sino de herramientas para que los padres resuelvan sus diferencias por una vía que no sea el conflicto violento. Establecen tres tipos de respuestas para afrontar las violencias y encaminarse hacia la paz: 1) solución no violenta de conflictos; 2) educación para la paz y los derechos humanos, y 3) procesos de 
reconciliación y mecanismos sociales. En este sentido, la paz no solamente es la ausencia de guerra, sino crear un ambiente libre de tensiones y de conflictos y propiciar que las personas desarrollen capacidades para lograr un desarrollo integral individual y colectivo, lo que implica alcanzar un estado de armonía en sus relaciones y desarrollar un adecuado manejo de conflictos.

Se busca una recuperación colectiva de la violencia a través de una "cultura de sanación" para superar los efectos de la violencia en Ciudad Juárez. Danesh y Clarke-Habibi (2007) mencionan que, en una cultura de la sanación, el alivio sicológico y la recuperación social del impacto destructivo de la violencia y la guerra en individuos y comunidades tiene lugar dentro de procesos tripartitos de adquisición de conocimiento, formación de relaciones y transformación de conductas.

Cuarto eje: prácticas restaurativas. En el caso del CFIC se han realizado principalmente a nivel familiar. Los conceptos de prácticas restaurativas y de justicia restaurativa se refieren a distintos tipos de procesos. Para Wachtel (2013), el término de prácticas restaurativas incluye el uso de procesos informales y formales que anteceden a las conductas indebidas, que crean proactivamente reacciones y crean un sentido de comunidad para evitar conflictos y conductas indebidas. La justicia restaurativa es reactiva y consta de respuestas formales o informales al delito y otras conductas indebidas, una vez que éstas ocurren. De esta manera, considera a la justicia restaurativa como un subgrupo de las prácticas restaurativas. Sostiene que, cuando el capital social, como red de relaciones, ya está bien establecido, es más fácil responder de manera efectiva a los actos indebidos y restaurar el orden social. Este autor señala que la función fundamental de las prácticas restaurativas es restaurar y forjar relaciones y que sus aplicaciones son útiles para reducir el crimen y la violencia, el hostigamiento escolar, fortalecer a la sociedad civil, restaurar relaciones y reparar el daño.

En general, los estudios sobre prácticas restaurativas se refieren a cómo éstas han sido aplicadas en contextos educativos. Gregory, Clawson, Davis y Gerewitz (2016) aplicaron prácticas restaurativas en el salón de clase con el objetivo de transformar las relaciones entre maestros y estudiantes y lograr equidad en la 
disciplina escolar. Los autores señalan los elementos que conformaron las prácticas restaurativas y las dividieron en dos grandes dimensiones: Prevención (construir relaciones y desarrollar comunidad) e Intervención (reparación de daños y restauración de la comunidad). La primera dimensión está compuesta por seis factores: afirmaciones afectivas, círculos proactivos, proceso justo, enfoque de comunidad restaurativa con el personal y las familias de los estudiantes y comprensión de hipótesis fundamentales. La segunda dimensión, la de intervención, está conformada por cinco factores: preguntas restaurativas, círculos receptivos, pequeños círculos improvisados, círculos restaurativos como conferencia y manejo reintegrativo de la vergüenza.

Mirsky (2011) analiza las prácticas restaurativas en el contexto escolar, mostrando cómo se establece este tipo de prácticas entre educadores y estudiantes. Él explora la filosofía y el proceso de las prácticas restaurativas y sostiene que construyen comunidad tanto en el salón de clases como en las escuelas, en su organización y lugares de trabajo, presentándose como una alternativa a las políticas excluyentes y punitivas de "tolerancia cero", que se mantienen en las escuelas. La hipótesis fundamental es que los seres humanos felices son más cooperativos y productivos, $\mathrm{y}$ es más probable que hagan cambios positivos en su conducta, y los que tienen una posición de autoridad hagan cosas con ellos, más que para ellos. Presenta historias relacionadas que muestran cómo se utiliza en ambientes concretos. Las prácticas restaurativas favorecen la comunicación más abierta, mejoran las relaciones interpersonales y así promueven un mejor clima, disciplina y seguridad en la escuela.

Quinto eje: prevención del suicidio. De acuerdo con el Instituto Nacional de Estadística y Geografía (INEGI, 2017), las entidades federativas que tuvieron mayores tasas de suicidio en 2015 por cada 100000 habitantes, fueron Chihuahua (11.4), Aguascalientes (9.9), Campeche (9.1) y Quintana Roo (9.1). En un estudio realizado por Esparza (2015), resultado de una colaboración entre la Universidad Autónoma de Ciudad Juárez, Doctorado en Psicología con Énfasis en Salud y Violencia y el CFIC, evaluó dos tipos de variables relacionadas con el suicidio a 1157 jóvenes de nivel Bachillerato en Ciudad Juárez: si la persona ha pensado en 
quitase la vida y si la persona ha intentado quitarse la vida. Las respuestas afirmativas para ambas preguntas presentan un porcentaje alto: $29 \%$ reporta haber pensado en quitarse la vida y $15 \%$ reporta haberlo intentado por lo menos una vez.

Castellví, Miranda, Parés, Almenara, Alonso, Blasco y Alonso (2017) realizaron un metaanálisis de estudios longitudinales cuyo objetivo fue evaluar la asociación y la magnitud del efecto de la exposición temprana a diferentes tipos de violencia interpersonal, con intento de suicidio y muerte por suicidio en jóvenes y adultos jóvenes. Se realizaron búsquedas en seis bases de datos hasta junio de 2015. Los criterios de inclusión fueron los siguientes: 1) evaluación de cualquier tipo de violencia interpersonal como factor de riesgo de intento de suicidio o suicidio: i) maltrato infantil [abuso físico, sexual, emocional, negligencia infantil], ii) bullying, (iii) violencia de pareja, y iv) violencia de la comunidad; 2) estudios de casos y controles de cohortes o casos basados en la población, y 3) sujetos de 12-26 años. Se usaron modelos aleatorios para metaanálisis. Concluyeron que la exposición temprana a la violencia interpersonal confiere un riesgo de intentos de suicidio y, en particular, de muerte por suicidio en jóvenes y adultos jóvenes. Recomiendan que las investigaciones futuras deberían abordar la efectividad para prevenir y detectar de forma temprana cualquier tipo de exposición a violencia interpersonal en edades tempranas.

Sexto eje: desarrollo humano y espiritualidad ignaciana. El Desarrollo Humano es un campo interdisciplinar de estudio y acción cuyo objetivo es la comprensión integral de las potencialidades, organización, procesos y relaciones de las personas y de los grupos humanos, para poder promover el desarrollo autónomo de los mismos. El Desarrollo Humano emplea como medio privilegiado la persona misma del promotor, su presencia y sus interacciones con los sujetos con los que trabaja (Documento base, 2002, p. 1). Para Opazo (2000) el gran desafío del desarrollo humano radica en comprender conceptualmente la contradicción que atraviesa nuestras sociedades, siendo que opone la racionalidad instrumental a la existencia vivida.

El concepto del PNUD (2015) acerca del Desarrollo Humano integra tres aspectos: por un lado, se centra en mejorar la vida 
de las personas, esto es, tener una vida larga y saludable, conocimientos y un nivel de vida digno. Por otro lado, el enfoque de oportunidades que consiste en crear las condiciones para el desarrollo humano, a través de la participación en la vida política y en la comunidad, sostenibilidad ambiental, seguridad humana y derechos e igualdad de género. Finalmente, el tercer aspecto es el de las posibilidades de elección, que enfatiza la libertad de las personas para elegir la vida que valoren.

De acuerdo con Palacio (2015), el mejor medio para alcanzar el desarrollo humano es la espiritualidad porque ésta permite que las personas contacten con lo mejor de sí mismos y puedan expandir su vida y potencialidades. La espiritualidad permite que las personas revaloren, resignifiquen y renueven su vida constantemente; por tanto, las personas siempre tienen la posibilidad de volver a empezar.

Cabarrús (2003) describe los aspectos básicos de la espiritualidad ignaciana, como lo son los ejercicios espirituales y sostiene que lo básico de ésta, está expresado en los verbos: experimentar, sentir, hacer, padecer y gozar. Plantea como objetivo hacer llegar la espiritualidad ignaciana a personas laicas, partiendo de que Ignacio de Loyola era laico cuando inició su proceso de conversión en Loyola. Cabarrús (2000, p. 10) menciona:

Era laico, cuando vivió la intensa experiencia de Manresa. Era laico, cuando experimentó y escribió los Ejercicios Espirituales. Era laico cuando empezó a tener junto a él compañeros a los que les fue dando los Ejercicios, y así, les fue comunicando un modo especifico de ser.

Los seis ejes mencionados anteriormente, más que ser entidades separadas, se interrelacionan, lo que se muestra en la figura 1, que da cuenta de la perspectiva multidimensional del trabajo del Centro. La delimitación conceptual entre las dimensiones es flexible y favorece la interacción que caracteriza las prácticas y las distintas estrategias de prevención y atención del CFIC. Cabe mencionar que estas dimensiones se dan en respuesta a las necesidades de la población, por lo que no es un modelo rígido, sino que es flexible y responde al diálogo constante de la institución con los distintos sectores sociales. 
FIGURA 1. Perspectiva multidimensional para la prevención y atención a la violencia Procesos

de duelo

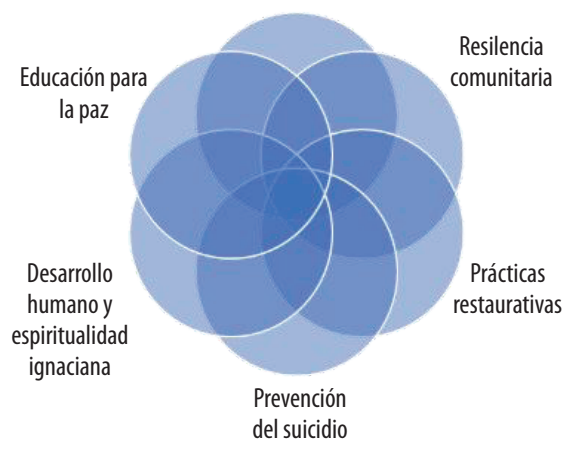

Fuente: elaboración propia.

\section{SISTEMATIZACIÓN DE LA PRÁCTICA: UNA RED DE EXPERIENCIAS Y RELACIONES}

El trabajo de sistematización tuvo como objetivo general comprender el proceso de reconstrucción del tejido social, a través de la sistematización de las experiencias y buenas prácticas de una asociación civil, desde la interacción de las dimensiones: procesos de duelo, resiliencia comunitaria, educación para la paz, prácticas restaurativas, desarrollo humano y espiritualidad ignaciana, para favorecer la comprensión de los procesos de cambio psicosociales en un contexto caracterizado por la pobreza y la violencia.

Durante la sistematización nos planteamos la pregunta de quiénes intervienen en el quehacer del CFIC y se mostró como un universo de relaciones (figura 2) que revela un abordaje sistémico y un dinamismo que va desde lo individual a lo social. Los Colaboradores son los profesionales del área de la salud mental que participan en las diferentes estrategias, entre las que destacan los talleres, los procesos psicoterapéuticos y los diplomados, entre otros. El CFIC cuenta con un Consejo Directivo que tiene distintas funciones como garantizar que el Centro cuente con suficientes recursos y se administren eficientemente, garantizar la planificación eficaz, evaluar su desempeño y mantener la transparencia, entre otros. 
FIGURA 2. Redes y estrategias de prevención e intervención psicosocial del CFIC

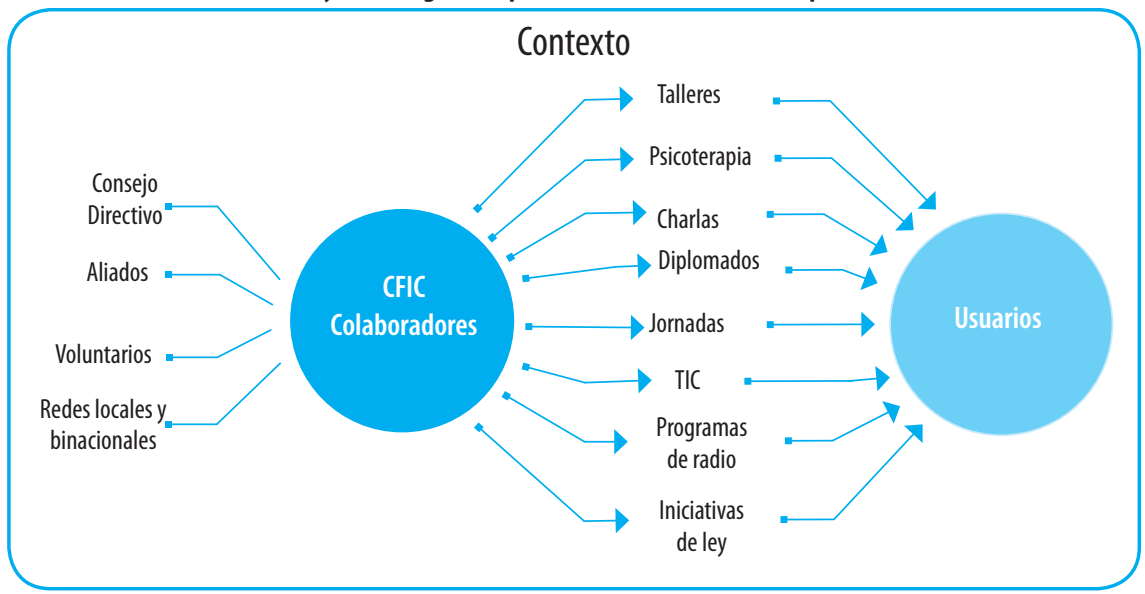

Fuente: elaboración propia.

De especial relevancia es el papel que juegan los Aliados tanto en el funcionamiento de la asociación como en posibilitar su inserción en distintos escenarios de Ciudad Juárez. Estas alianzas se refieren a las uniones entre los distintos grupos que tienen como objetivo lograr un fin común que, en este caso, es la reconstrucción del tejido social. Entre los aliados se destaca tanto el papel de las Fundaciones como el del Sector Público, que apoyan económicamente las distintas estrategias de intervención. Entre las fundaciones están: la Fundación del Empresariado Chihuahense (Fechac), el Fideicomiso para la Competitividad y Seguridad Ciudadana (Ficosec) Juárez y los Supermercados $S^{*}$ Mart. En el Sector Público, cuentan con el apoyo del Sistema Nacional para el Desarrollo Integral de la Familia (DIF) del estado, la Dirección General de Desarrollo Social del Municipio de Ciudad Juárez y el Programa Nacional de Prevención del Delito.

También tienen alianzas con universidades e institutos que les permiten brindar una oferta educativa conjunta, la evaluación de impacto de sus programas y fortalecer la profesionalización de sus colaboradores. Algunos de estos aliados son: la Universidad Autónoma de Ciudad Juárez, la Universidad Iberoamericana, Fortalessa, el Instituto Hispanoamericano de Suicidología y la Comunidad Latinoamericana de Resiliencia. Cabe mencionar 
que también tienen aliados que posibilitan los enlaces con distintos sectores de la población como son iglesias, escuelas, hospitales públicos y autoridades municipales y estatales. Uno de los recursos más importantes del CFIC es la extensa colaboración en redes locales y binacionales, por el interés de las ciudades fronterizas para construir estrategias comunes de prevención e intervención psicosocial que atiendan la complejidad de las necesidades de las poblaciones en ambos lados de la frontera.

Las estrategias utilizadas también son múltiples; desde abordajes individuales, de pareja, familiares, grupales, hasta el uso de medios de comunicación para un sector de la población más extenso, así como de las tecnologías de información y comunicación, de programas de radio diarios que ofrecen distintos contenidos asociados a los seis ejes ya mencionados y su página en Facebook. Los diplomados que imparten, generalmente están vinculados a universidades e institutos. En las jornadas abordan anualmente temáticas que den respuesta a necesidades emergentes de la entidad y convocan a profesionales e investigadores para analizar los aspectos teóricos y de intervención actuales sobre esos temas de interés. También han favorecido, junto con legisladores aliados, propuestas de iniciativas de ley vinculadas con el divorcio contencioso (política pública estatal) y la prevención del suicidio (política pública nacional). De esta manera, las personas que participaron en la sistematización fueron los colaboradores del Centro a través de relatos de vida y testimoniales, y líneas del tiempo (mapeo histórico), o entrevistas y grupos de discusión con los usuarios y los aliados.

Los usuarios entrevistados fueron niños, adolescentes y adultos que se encuentran en distintas poblaciones de la frontera mexicana, que son ámbitos de intervención del CFIC: Ciudad Juárez (en algunas Zonas de Atención Prioritaria), Villas de Salvárcar, el Valle de Juárez, ubicado en las márgenes del Río Bravo, específicamente, en Guadalupe, Praxedis G. Guerrero y San Isidro, estas últimas localidades con un alto índice de migración de los habitantes hacia ciudades estadounidenses por la violencia dirigida contra ellos. También han ofrecido talleres a la comunidad rarámuri y capacitación en su modelo de intervención a colaboradores y usuarios de la Arquidiócesis de Tabasco. 
De esta manera, para dar cuenta de la complejidad de las prácticas y experiencias significativas en este contexto fronterizo se utilizó la narrativa como método de investigación, que permite la construcción, deconstrucción y reconstrucción de las historias. Para Josselson (2011), la investigación narrativa busca la interpretación a partir de la articulación de subjetividades de investigadores y participantes que tiene como objetivo explorar y conceptualizar la experiencia humana que se representa en forma textual (ya sea oral o escrita). Para Cabruja, Íniguez y Vázquez (2000), la construcción del mundo social con base en significados se fundamenta, entre otros aspectos, en la intersubjetividad que entiende que los significados se construyen en las relaciones, ya que las personas actuamos en función de otras en relación con contextos, significados y producciones sociales, como las instituciones, costumbres, discursos y prácticas.

\section{COMENTARIOS FINALES}

En primer término, la sistematización de prácticas y experiencias significativas muestra, potencia y visibiliza una serie de estrategias vinculadas con la prevención y la atención psicosocial dirigida a personas con necesidades asociadas a las consecuencias de la violencia en la población. También favorece el proceso de reflexión, de problematización, de nombrar las experiencias y comprenderlas como un proceso histórico y situado de un grupo de trabajo sobre su quehacer, y que puede generar estrategias de fortalecimiento de nuevas formas de acción.

Resultan evidentes los planteamientos innovadores en la integración de los distintos abordajes que dan lugar a una visión y prácticas multidimensionales que caracterizan el quehacer de la institución, desde las seis dimensiones mencionadas, cuya emergencia ha respondido a situaciones propias del contexto en el que se desarrollan: procesos de duelo, resiliencia comunitaria, educación para la paz, prácticas restaurativas, prevención del suicidio, desarrollo humano y espiritualidad ignaciana, que apuntan ya a mostrar la complejidad de las experiencias y del contexto. El contexto evidentemente no es lo que le ocurre a los otros, ya que forma parte de la trama de la vida cotidiana de los habitantes de 
esa población en cualquiera de los círculos en los que se encuentren, en este caso, de todos los actores sociales que participan en el quehacer de la institución. De esta manera, los escenarios y las acciones humanas tienen sentido en marcos sociales que permitan comprender las experiencias y las elaboraciones y reelaboraciones de significados. Así, el contexto forma parte de la realidad construida en las narraciones.

Finalmente, cabe destacar que el trabajo del CFIC se caracteriza por generar efectos multisistémicos que van de lo individual hacia lo colectivo y viceversa. Esta forma de trabajar desde una perspectiva multidimensional y multisistémica responde al reconocimiento de la violencia como un fenómeno complejo en cuyo entramado se encuentran aspectos individuales, familiares/relacionales, institucionales, comunitarios y socioculturales; es por ello que se requiere de la consideración de una amplia gama de estrategias e intervenciones que sean capaces de incidir en estos distintos niveles y que, por tanto, puedan participar en la recons-

250 trucción del tejido social.

\section{REFERENCIAS BIBLIOGRÁFICAS}

Álvarez, E. y González, I.N. (2013). Derechos humanos, ciudadanía y paz. Construcción de la democracia en México. México: Cátedra Eusebio Francisco Kino, S. J.

Armour, M. (2003). Meaning Making in the Aftermath of Homicide, Death Studies, 27(6), 519-540. doi: 10.1080/07481180302884.

Barnechea, M.M. y Morgan, M.L. (2010). La sistematización de experiencias: la producción de conocimientos desde y para la práctica. Tendencias y Retos, 15, 97-107.

Cabarrús, R. (2003). La espiritualidad ignaciana, es laical. Apuntes sobre "ignacianidad". Guatemala: Universidad Rafael Landívar.

Cabruja, T., Íñiguez, L. y Vázquez, F. (2000). Cómo construimos el mundo: relativismo, espacios de relación y narratividad. Anàlisi, 25, 61-94. Recuperado de http://ddd.uab. es/pub/analisi/02112175n25p61.pdf 
Castellví, P., Miranda-Mendizábal, A., Parés-Badell, O., Almenara, J., Alonso, I., Blasco, M.J., y Alonso, J. (2017). Exposure to violence, a risk for suicide in youths and young adults. A meta-analysis of longitudinal studies. Acta Psychiatrica Scandinavica, 135(3), 195-211. doi: 10.1111/ acps. 12679 .

Centro Familiar para la Integración y Crecimiento, A.C. Consultado el 28 de agosto de 2016. Recuperado de http:// cficcdjuarez.org/

Corona, S. y Kaltmeir, O. (2012). Introducción. En S. Corona y O. Kaltmeir (Eds.). En diálogo. Metodologias horizontales en Ciencias Sociales y Culturales (pp.11-24). Barcelona, España: Gedisa.

Crispín, M.L. y Ruiz, M. (2010). Huellas de un caminar. Misión Jesuita de Bachajón. México: Universidad Iberoamericana.

Danesh, H.B. y Clarke-Habibi, S. (2007). Education for Peace Curriculum Manual: A Conceptual And Practical Guide. Vol. 1. Vancouver, Canadá: EFP.

De Souza, J. F. (2008). Sistematización: Un instrumento pedagógico en los proyectos de desarrollo sustentable. Magisterio. Educación y Pedagogía, 33, 8-13.

Documento base (2002). Mancillas, C.; Martínez, L.B.; Ortega, L.; Piña, L.; Plasencia, M.; Segrera, A.S.; Vergara, L. Fundamentos académicos y profesionales del Desarrollo Humano en la Universidad Iberoamericana. México: Universidad Iberoamericana.

Dreyer, Y. (2015). Community Resilience and Spirituality: Keys to Hope for a Post-Apartheid South Africa. Pastoral Psychology, 64 (5), 651-662. https://doi.org/10.1007/ s11089-014-0632-2

Escobedo, J. (2014). Escuelas de perdón y reconciliación: Hacia una cultura politica de perdón y reconciliación. (Tesis doctoral). Universidad Iberoamericana, México.

Esparza, O.A., Gutiérrez, M., Montañez, P. y Carrillo, I.C. (2016). Validity of a paranoid thoughts due to social violence scale in Juarez Mexico. European Scientific Journal, Special edition, 28-36. 
Esparza, O.A. (2015). Evaluación cuantitativa y cualitativa del Programa "Resiliencia comunitaria". Ciudad Juárez, México: Universidad Autónoma de Ciudad Juárez/Centro Familiar para la Integración y Crecimiento.

Ghiso, A.M. (1998). De la práctica singular al diálogo con lo plural. Aproximaciones a otros tránsitos y sentidos de la sistematización en épocas de globalización. Recuperado de http://unpan1.un.org/intradoc/groups/public/documents/icap/unpan033101.pdf

Gillies, J., y Neimeyer, R.A. (2006). Loss, Grief, and the Search for Significance: Toward a Model of Meaning Reconstruction in Bereavement, Journal of Constructivist Psychology, 19(1), 31-65. doi: 10.1080/10720530500311182

Gregory, A., Clawson, K., Davis, A., y Gerewitz, J. (2016). The Promise of Restorative Practices to Transform TeacherStudent Relationships and Achieve Equity in School Discipline, Journal of Educational and Psychological Consultation, 26(4), 325-353. doi: 10.1080/10474412.2014.929950

Instituto Nacional de Estadística y Geografía (INEGI) (2017). Estadísticas a propósito del día mundial para la prevención del suicidio. Recuperado de http://www.inegi.org. $\mathrm{mx} /$ saladeprensa/aproposito/2017/suicidios2017_Nal.pdf

Jara, O. (1998). Para sistematizar experiencias. San José: AlFORJA Josselson, R. (2011). Narrative Research. Constructing, Deconstructing and Reconstructing Story. En F. J. Wertz, K. Charmaz, L.M. McMullen, R. Josselson,, R. Anderson y E. McSpadden (Eds.). Five Ways of Doing Qualitative Analysis. Phenomenological Psychology, Grounded Theory, Discourse Analysis, Narrative Research and Intuitive Inquiry (pp. 224-242). Nueva York: Guilford.

Lima, A. y Lima, M. (2014). Cuarto informe. Crímenes en Juárez 2009 y homicidios 2008-2012. Ciudad Juárez, México: Universidad Autónoma de Ciudad Juárez, Observatorio de Violencia Social y de Género/BENMA.

Madsen, W. y O'Mullan, C. (2016). Perceptions of Community Resilience After Natural Disaster in a Rural Australian Town. Journal of Community Psychology, 44(3), 277-292. doi.org/10.1002/jcop.21764 
Martínez, W. (2012). Situación y evolución demográfica. En L. Barraza y H. Almada (2012). La realidad social y las violencias. Ciudad Juárez. Diagnóstico sobre la realidad social, económica y cultural de los entornos locales para el diseño de intervenciones en materia de prevención y erradicación de la violencia (pp. 21-52). México: Universidad Autónoma de Ciudad Juárez y Comisión Nacional para prevenir y erradicar la violencia contra las mujeres (CONAVIM).

Meza, E.; García, S.; Torres, A.; Castillo, L.; Sauri, S. y Martínez, B. (2008). El proceso de duelo: un mecanismo humano para el manejo de las pérdidas emocionales. Revista de Especialidades Médico-Quirúrgicas, 13(1), 28-31.

Mirsky, L. (2011). Restorative Practices: Giving Everyone a Voice to Create Safer Saner School Communities. The Prevention Researcher, 18(5) 3-6.

Opazo, A. (febrero de 2000). El sujeto del desarrollo humano. Cátedra "Paulo Freire". Ponencia en el VIII Simposium de Educación.Tlaquepaque, Jalisco, México.

Organización de las Naciones Unidas para la Alimentación y la Agricultura (FAO) (2015). Plantilla de buenas prácticas. Recuperado de http://www.fao.org/3/a-as547s.pdf

Organización Mundial de la Salud (OMS, 2004). Prevención de los Trastornos Mentales. Intervenciones efectivas y opciones de politicas. Informe compendiado. Ginebra: OMS.

Palacio, C.J. (2015). La espiritualidad como medio de desarrollo humano. Cuestiones Teológicas, 42(98), 459-481. Recuperado de https://revistas.upb.edu.co/index.php/cuestiones/article/view/6621

Palomar, J. (2015). Resiliencia, educación y movilidad social en adultos beneficiarios del Programa de Desarrollo Humano Oportunidades. México: Universidad Iberoamericana.

Pieck, E. (2012). La sistematización de experiencias significativas de formación para el trabajo de los ICAT. México: Universidad Iberoamericana.

Programa de las Naciones Unidas para el Desarrollo (PNUD) (2017). Sistematización para transferir conocimiento. Recuperado de http://portalsiget.net/archivosSIGET/publicaciones/Archivos/2382017_PNUD.pdf 
Programa de las Naciones Unidas para el Desarrollo (PNUD) (2015). ¿Qué es el desarrollo humano? Recuperado de http://hdr.undp.org/es/content/\%C2\%BFqu\%C3\%A9-esel-desarrollo-humano

Rodríguez, G., Gil, J. y García, E. (1999). Metodología de la investigación cualitativa. Granada: Aljibe.

Twigg, J. (2007). Caracteristicas de una comunidad resiliente ante los desastres. Recuperado de http://www.eird.org/wikies/ images/Spanish_Characteristics_disaster_high_res.pdf

Uriarte, J.D. (2013). La perspectiva comunitaria de la resiliencia. Psicología Politica, 47, 7-18.

Wachtel, T. (2013). Definiendo qué es Restaurativo. Pennsylvania: Instituto Internacional de Prácticas Restaurativas. Recuperado de http://www.iirp.edu/pdf/Defining-Restorative-Spanish.pdf. 